\title{
VTE prevention: Patient selection and treatment planning throughout pregnancy
}

\author{
While VTE is generally rare during pregnancy, a prior VTE, an inherited \\ thrombophilia, or another predisposing medical condition increase the risk. \\ Here, guidance on selecting patients for anticoagulation prophylaxis and \\ 3 dosing strategies.
}

\section{Julianne Lauring, MD}

\section{IN THIS} ARTICLE

VTE risk factors

page 41

Prophylaxis dosing options

page 42

\section{Postpartum}

chemoprophylaxis

page 44

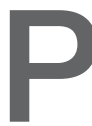

regnancy and the postpartum period are times of increased risk for venous thromboembolism (VTE). While VTE is a rare event overall, it is responsible for more than $9 \%$ of maternal deaths in the United States. ${ }^{1}$ The increased risk of VTE exists throughout pregnancy, rising in the third trimester. ${ }^{2}$ The highest-risk period is the first 6 weeks postpartum, likely peaking in the first 2 to 3 weeks and returning to baseline at about 12 weeks postpartum. ${ }^{2,3}$

To reduce this source of maternal harm, the National Partnership for Maternal Safety and the Council on Patient Safety in Women's Health Care recommend the use of VTE prevention bundles. Bundles include standard assessment of risk during prenatal care, any admission to the hospital, and postpartum coupled with standard recommendations for treatment. ${ }^{4-6}$ Multiple published guidelines

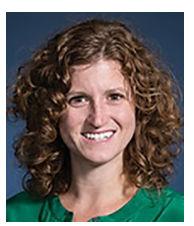

Dr. Lauring is Assistant Professor, Division of Maternal Fetal Medicine, Department of Obstetrics and Gynecology, University of Massachusetts Medical School, and Maternity Center Medical Director, UMass Memorial Health, Worcester.

The author reports no financial relationships relevant to this article.

doi: 10.12788/obgm.0116 are available for prevention of VTE in pregnancy, and they provide varying recommendations on patient selection and treatment. Many of these recommendations are based on low quality of evidence, making the choice of standard practice difficult.

In this article, I attempt to simplify patient selection and treatment based on currently published guidelines from the American College of Obstetricians and Gynecologists (ACOG), Royal College of Obstetricians and Gynaecologists (RCOG), American College of Chest Physicians (CHEST), American Society of Hematology (ASH), and expert opinion.

\section{Determining VTE risk and need for prophylaxis}

CASE 1 Woman with factor V Leiden

A 25-year-old woman (G1P0) presents for her initial prenatal visit. She says she is a carrier for factor $\mathrm{V}$ Leiden but has never had a clot. She was tested after her sister had a VTE. She asks, does she need VTE prophylaxis before her delivery?

What are the considerations and options for this patient?

\section{Options for VTE prophylaxis}

Before considering patients at risk for VTE, it is helpful to review the options for prophylaxis. 
Patients can undergo clinical surveillance or routine care with attention to VTE symptoms and a low threshold for workup.

There are 3 categories of chemoprophylaxis for prevention of VTE. (TABLE 1, page 42, offers examples of dosing regimens.) No strategy has been proven optimal over another:

- prophylactic-dose: the lowest, fixed dose.

- intermediate-dose: lacks a standard definition and is any dose higher than prophylactic-dose but lower than therapeutic-dose. This includes fixed twice-daily doses, weight-based doses, and incrementally increasing doses.

- therapeutic-dose: typically used for treatment but mentioned here since patients with high-risk conditions may use it for prevention of VTE.

The preferred agent for VTE chemoprophylaxis is low molecular weight heparin (LMWH; dalteparin, enoxaparin). LMWH has a lower risk of complications than unfractionated heparin (UFH) and can be injected once daily. LMWH and UFH do not cross the placenta. LMWH and UFH are safe in breastfeeding. Oral direct thrombin inhibitors and anti-Xa inhibitors are not recommended in pregnancy or lactation at this time. Warfarin is avoided in pregnancy except in situations with mechanical heart valves, which will not be addressed here. Patients taking warfarin for long-term anticoagulation can transition back while breastfeeding with appropriate bridging.

Expert opinion recommends antepartum chemoprophylaxis when there is a $2 \%$ to $3 \%$ risk of VTE in pregnancy. ${ }^{7-9}$ This is balanced against an approximately $2 \%$ overall risk of bleeding, with less than $1 \%$ risk of bleeding antepartum. ${ }^{9}$

\section{Risk factors for VTE}

History of VTE. The most important risk factor for VTE is a personal history of prior VTE. ${ }^{6}$ Recurrence risks have been widely reported and depend on the factors surrounding the initial event. For patients with a prior provoked deep vein thrombosis (DVT; associated with trauma or surgery), the antepartum VTE risk likely is less than 1\%, and VTE chemoprophylaxis is not recommended antepartum. ${ }^{7}$

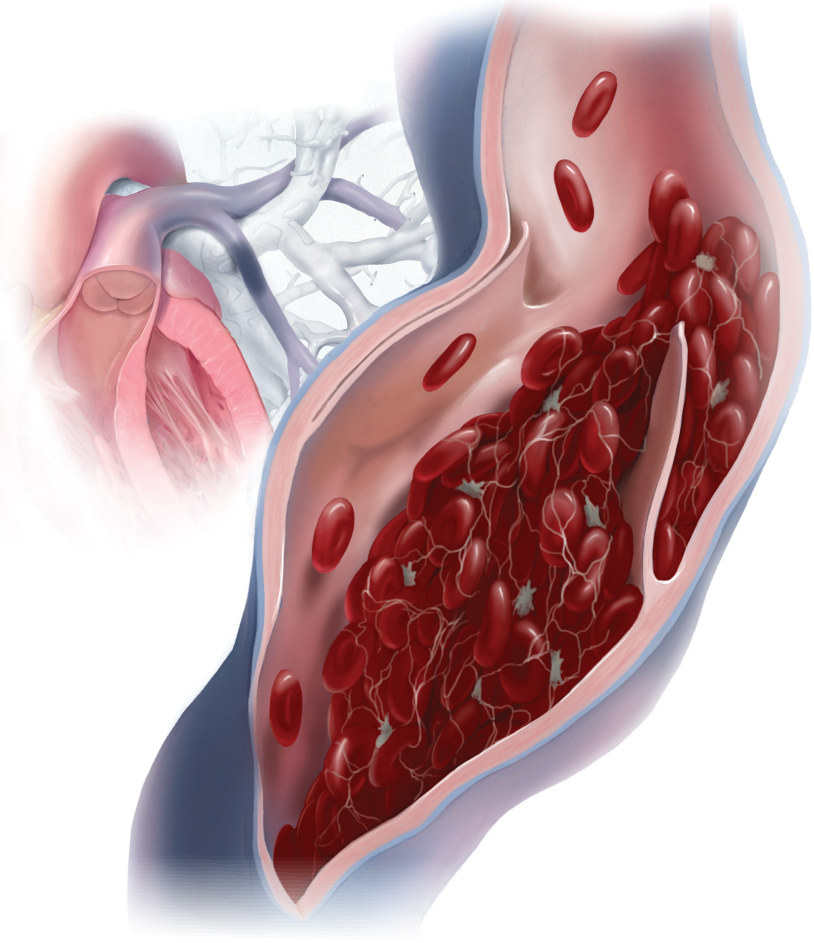

For patients with a prior VTE that was not associated with surgery or trauma (unprovoked), the risk is approximately $3 \%$; for prior VTE related to pregnancy or hormonal contraception, the risk is approximately $6 \%{ }^{7}$ For both of these groups, prophylactic-dose antepartum is recommended. Patients with recurrent VTE are often taking long-term anticoagulation. Anyone on long-term anticoagulation should be placed on therapeuticdose antepartum. For patients not receiving long-term anticoagulation, consider a hematology consultation when available, and begin an intermediate-dose or therapeuticdose regimen after assessing other risk factors and the risk of bleeding and discussing treatment with the patient.

Thrombophilias. The next most important risk factor is the presence of inherited thrombophilias. ${ }^{6}$ Factor V homozygote, prothrombin G20210A mutation homozygote, antithrombin deficiency, and combined factor V heterozygote and prothrombin G20210A heterozygote (also called compound heterozygote) have the strongest association with VTE in pregnancy. ${ }^{8}$ It is recommended that patients with these high-risk thrombophilias receive prophylactic-dose antepartum. ${ }^{8}$

Factor $\mathrm{V}$ heterozygote, prothrombin G20210A mutation heterozygote, and protein
FAST

TRACK

The most important

risk factor for

VTE is a personal

history of prior

VTE; the next

most important

risk factor is

the presence

of inherited

thrombophilias 
TABLE 1 Dosing options for VTE prophylaxis

\begin{tabular}{|c|c|}
\hline \multicolumn{2}{|l|}{ Prophylactic-dose } \\
\hline & Enoxaparin $40 \mathrm{mg}$ daily \\
\hline & Dalteparin $5,000 \mathrm{U}$ daily \\
\hline & UFH 5,000 U daily \\
\hline \multicolumn{2}{|l|}{ Intermediate-dose } \\
\hline \multirow{3}{*}{$\begin{array}{l}\text { No standard dose, other dosing } \\
\text { regimens exist }\end{array}$} & Enoxaparin $0.5 \mathrm{mg} / \mathrm{kg}$ daily or enoxaparin $40 \mathrm{mg}$ every $12 \mathrm{~h}$ \\
\hline & Dalteparin $5,000 \mathrm{U}$ every $12 \mathrm{~h}$ \\
\hline & $\begin{array}{l}\text { UFH 5,000 } \mathrm{U} \text { every } 12 \mathrm{~h} \text { in the first trimester; } 7,500 \mathrm{U} \text { every } 12 \mathrm{~h} \text { in } \\
\text { the second trimester; } 10,000 \mathrm{U} \text { every } 12 \mathrm{~h} \text { in the third trimester }\end{array}$ \\
\hline \multicolumn{2}{|l|}{ Therapeutic-dose } \\
\hline & Enoxaparin $1 \mathrm{mg} / \mathrm{kg}$ every $12 \mathrm{~h}$ or $1.5 \mathrm{mg} / \mathrm{kg}$ daily \\
\hline & Dalteparin $100 \mathrm{U} / \mathrm{kg}$ every $12 \mathrm{~h}$ or $200 \mathrm{U} / \mathrm{kg}$ daily \\
\hline & UFH $10,000 \mathrm{U}$ twice daily adjusted to therapeutic aPTT levels \\
\hline
\end{tabular}

\section{FAST}

TRACK

Patients with lowrisk thrombophilias and no personal history of VTE or first-degree relative with VTE can be monitored with clinical surveillance antepartum
C or protein S deficiency are considered lowrisk thrombophilias. Patients with low-risk thrombophilias and no personal history of VTE or first-degree relative with VTE can be monitored with clinical surveillance antepartum. However, if a family history of VTE or other risk factors for VTE are present, antepartum prophylactic-dose is recommended. Clinical factors to consider antepartum include obesity, age older than 35 years, parity of 3 or higher, varicose veins, immobility, smoking, assisted reproductive technology use, multiple gestation, and preeclampsia. ${ }^{10}$

Antiphospholipid syndrome (APS) is another high-risk condition. For patients not taking long-term anticoagulation antepartum, prophylactic-dose is recommended. For patients on long-term anticoagulation, therapeutic-dose is recommended.

Other medical conditions. Patients with medical conditions that place them at high risk for VTE may warrant prophylactic-dose antepartum. These include active cancer, active systemic lupus erythematosus, sickle cell disease, nephropathy, and inflammatory bowel disease. ${ }^{10}$ This decision can be made in conjunction with other specialists caring for the patient.

Antepartum prophylactic-dose is not recommended for low-risk patients as there is less than 1\% risk of VTE. ${ }^{7}$ (TABLE 2, page 44, summarizes antepartum chemoprophylaxis recommendations.)

CASE 1 continued Patient develops another VTE risk factor

The patient is being followed with clinical surveillance. At 19 weeks' gestation, she presents to the emergency department with shortness of breath and fever. She is diagnosed with COVID-19 and is admitted by a medicine service. They call the $\mathrm{OB}$ team to ask for recommendations regarding anticoagulation.

What should the next steps include?

\section{Hospitalization and nonobstetric surgery} are risk factors for VTE. Many hospitals use a standardized assessment for all inpatients, such as the Padua or Caprini VTE risk assessment scores. These can be modified for use in pregnant patients, although neither scoring system is currently validated for use in pregnancy. ${ }^{5}$ For any pregnant patient admitted to the hospital, mechanical prophylaxis is recommended.

COVID-19. Infection with the novel severe acute respiratory syndrome coronavirus 2 (SARS-CoV-2) and its associated clinical syndrome, COVID-19, is associated with increased rates of VTE. Recommendations for pregnant patients with COVID-19 are the same as for the general population. During hospitalization for COVID-19, pregnant patients should be placed on prophylacticCONTINUED ON PAGE 44 
TABLE 2 Recommendations for antepartum chemoprophylaxis

\begin{tabular}{|c|c|c|}
\hline \multicolumn{3}{|c|}{ Antepartum } \\
\hline Prophylactic-dose & Therapeutic-dose & Clinical surveillance \\
\hline $\begin{array}{l}\text { - Prior VTE that } \\
\text { was unprovoked } \\
\text { or associated } \\
\text { with hormonal } \\
\text { contraception, } \\
\text { not currently on } \\
\text { anticoagulation } \\
\text { - Factor V homozygote } \\
\text { - Prothrombin G20210A } \\
\text { mutation homozygote } \\
\text { - Compound } \\
\text { heterozygote } \\
\text { - Antithrombin } \\
\text { deficiency }\end{array}$ & $\begin{array}{l}\text { Currently on } \\
\text { long-term } \\
\text { anticoagulation } \\
\text { Thrombophilia with } \\
\text { recurrent VTE }\end{array}$ & $\begin{array}{l}\text { - } \text { Factor V } \\
\text { heterozygote } \\
\text { - Prothrombin } \\
\text { G20210A mutation } \\
\text { heterozygote } \\
\text { - Protein C deficiency } \\
\text { - Protein S deficiency } \\
\text { - Provoked VTE not } \\
\text { related to pregnancy } \\
\text { or hormonal } \\
\text { contraception } \\
\text { - Low-risk patients }\end{array}$ \\
\hline
\end{tabular}

Abbreviations: VTE, venous thromboembolism.

dose chemoprophylaxis. Patients should not be discharged home on chemoprophylaxis, and patients managed as outpatients for their disease do not need chemoprophylaxis. ${ }^{11}$

Management approach. Prophylacticdose administration is recommended during hospital stay for all patients admitted with anticipated length of stay of 3 days or longer and who are not at high risk for bleeding or delivery. ${ }^{10}$ Both LMWH and UFH are options for inpatients. For any nonobstetric surgery or admission, LMWH may be most appropriate. However, as most obstetrics admissions are at increased risk for delivery, UFH 5,000 U twice daily to 3 times daily is the best option to increase the chances for neuraxial anesthesia. (I review anesthesia considerations for delivery later in this article.) For patients at high risk for bleeding or delivery, mechanical prophylaxis alone, with elastic stockings or pneumatic compression devices, can be used.

\section{CASE 1 continued Patient is discharged} home

The patient received enoxaparin while she was in the hospital. She is now discharged and doing well. She asks, will she need anticoagulation prophylaxis after delivery?

How would you counsel her?

\section{Chemoprophylaxis in the postpartum period}

With no risk of fetal harm and a higher risk of VTE per day, the threshold for chemoprophylaxis is lower in the postpartum period. The risk of postpartum bleeding is less than $1 \%$, with the most common complication being wound hematomas $(0.61 \%) .{ }^{9}$ For this case patient, the COVID-19 diagnosis does not alter the recommendations for postpartum chemoprophylaxis. Additionally, as the need for neuraxial anesthesia has passed, the use of intermediate-dose chemoprophylaxis over prophylactic-dose is advocated in the postpartum period, especially in obese patients. ${ }^{12}$

As mentioned previously, there is no standard definition of intermediate-dose. Data suggest that a weight-based intermediate-dose is most likely to achieve therapeutic levels of anti-Xa in this high-risk population compared with a fixed dose. ${ }^{13,14}$ For example, enoxaparin $0.5 \mathrm{mg} / \mathrm{kg}$ twice daily is recommended for patients with class 3 obesity or higher by the Society for Maternal-Fetal Medicine. ${ }^{12}$

As a rule, anyone who was on chemoprophylaxis antepartum should be continued on at least an equivalent dose for 6 weeks postpartum. Postpartum, patients with any prior DVT should take prophylactic-dose or intermediate-dose chemoprophylaxis for 6 weeks. Patients with a known high-risk thrombophilia should receive prophylactic-dose or intermediate-dose chemoprophylaxis postpartum for 6 weeks. For patients with a lowrisk thrombophilia, prophylactic-dose or intermediate-dose chemoprophylaxis is recommended for 6 weeks.

For low-risk patients without prior VTE or thrombophilia, standardized risk assessment is recommended.

\section{Cesarean delivery}

Cesarean delivery (CD) is a risk factor for postpartum VTE. ${ }^{9}$ A universal chemoprophylaxis strategy has not been proven in this patient population. Mechanical prophylaxis 
TABLE з Recommendations for postpartum chemoprophylaxis

\begin{tabular}{l|c|c}
\hline \multicolumn{1}{c}{ Prophylactic-dose } & Postpartum \\
\hline \multicolumn{1}{c}{ or intermediate-dose if obese } & Therapeutic-dose & Clinical surveillance \\
\hline - Prior VTE & $\bullet$ Currently on long-term anticoagulation & Low-risk patients without multiple \\
- Factor V homozygote & & \\
- Prothrombin G20210A mutation \\
homozygote \\
- Compound heterozygote \\
- Antithrombin deficiency \\
- Factor V heterozygote \\
- Prothrombin G20210A mutation \\
heterozygote \\
- Protein C deficiency \\
- Protein S deficiency \\
- Patients with multiple risk factors \\
based on a risk assessment model
\end{tabular}

with sequential compression devices is recommended for all patients undergoing CD pre-procedure and until patients are fully ambulatory. ${ }^{8,9}$ Early ambulation also should be encouraged.

Many risk assessment models are available for postoperative VTE prevention, and they have widely different chemoprophylaxis rates. Studies have shown chemoprophylaxis rates of $85 \%$ by RCOG, $1 \%$ by ACOG, $35 \%$ by CHEST, $94 \%$ by Caprini, and less than $1 \%$ by Padua. ${ }^{15,16}$ In addition to the antepartum patient-specific risk factors mentioned, postpartum risk factors include infection, postpartum hemorrhage, and transfusion. Based on data extrapolated from the nonobstetric literature, chemoprophylaxis is recommended until discharge from the hospital unless risk factors are expected to continue. ${ }^{9}$

\section{Neuraxial anesthesia}

For patients who require postpartum chemoprophylaxis, the Society for Obstetric Anesthesia and Perinatology (SOAP) offers evidence-based guidelines for use after neuraxial anesthesia. UFH can be initiated 1 hour or longer after a neuraxial procedure and
1 hour or longer after catheter removal. Prophylactic-dose LMWH can be restarted at 12 hours or longer after a neuraxial procedure and at 4 to 6 hours or longer after catheter removal. For patients restarting intermediate-dose or therapeutic-dose, the recommendations are to wait 24 hours or longer after a neuraxial procedure and 4 hours or longer after catheter removal. ${ }^{17}$ Timing can be individualized based on the patient's risk of hemorrhage and surgical bleeding. Although it may be tempting to delay chemoprophylaxis in the setting of bleeding, postpartum hemorrhage and transfusion increase the risks of VTE. In this setting, it is best to consider the use of UFH, which safely can be started earlier than LMWH.

For patients without neuraxial anesthesia, ACOG recommends chemoprophylaxis 4 to 6 hours after vaginal delivery and 6 to 12 hours after CD. ${ }^{8}$ (TABLE 3 summarizes recommendations for postpartum chemoprophylaxis.)

\section{Adjusting the anticoagulation regimen \\ CASE 2 Pregnant woman with prior VTE}

A 36-year-old woman (G1P0) with prior VTE is 
taking enoxaparin $40 \mathrm{mg}$ daily. She asks, does she need any blood work for her anticoagulation?

What would you test for?

Increased renal clearance of LMWH and increased volume of distribution during pregnancy has led to the consideration of monitoring anti-Xa levels. There are no published standards or recommendations for dose adjustment. At this time, anti-Xa level monitoring antepartum is not recommended, but it may be considered when a patient is at the extremes of weight. With a weight-based strategy in the postpartum period, monitoring is not recommended as studies show a higher likelihood of therapeutic anti-Xa levels with this approach. ${ }^{13,14}$ This is an active area of research, and these recommendations may change.

For prophylactic-dose or intermediatedose anticoagulation, a peak anti-Xa level of 0.2 to $0.6 \mathrm{U} / \mathrm{mL}$ is generally accepted as the target. For therapeutic-dose, a peak anti-Xa level of 0.6 to $1.2 \mathrm{U} / \mathrm{mL}$ is generally accepted as the therapeutic range. This blood draw must be collected 4 hours after the third dose.

\section{FAST}

At this time, anti-Xa level monitoring antepartum is not recommended, but it may be considered when a patient is at the extremes of weight

\section{CASE 2 continued Anticoagulation}

considerations nearing delivery

The patient is now at 36 weeks' gestation, and she asks, what should be done regarding her anticoagulation prior to delivery?

What would be an appropriate approach? Traditionally, patients were transitioned to UFH at 36 weeks and allowed to present in spontaneous labor to increase the likelihood of neuraxial anesthesia. The alternative is to continue prophylactic-dose LMWH until a scheduled delivery. While the SOAP guidelines establish the timeframe that is safe to proceed with neuraxial anesthesia, there is variation in practice, so consider discussing this with your anesthesia providers.

SOAP considers prophylactic-dose UFH to be 5,000 U 2 to 3 times per day. In this setting, neuraxial anesthesia can be placed more than 4 to 6 hours from the last dose. ${ }^{17}$ But due to the pharmacokinetics of pregnancy, ACOG recommends $10,000 \mathrm{U}$ in the third trimester. ${ }^{8}$ This dose is considered intermediate-dose by SOAP, and 12 hours or longer plus a normal activated partial thromboplastin time (aPTT) or undetectable anti-Xa level are required prior to neuraxial anesthesia. This is the same time allowed for prophylactic-dose LMWH without lab work. Prophylactic-dose LMWH is considered to be enoxaparin $40 \mathrm{mg}$ or less daily or $30 \mathrm{mg}$ twice daily, and dalteparin 5,000 U daily. For therapeutic-dose LMWH or UFH, 24 hours or more from last dose is recommended prior to neuraxial anesthesia. For intermediatedose $\mathrm{LMWH}$, data are limited to recommend anything between 12 and 24 hours. ${ }^{17}$

In my practice, we favor a shared decisionmaking approach with patients. We discuss the likelihood of labor prior to 39 weeks based on a patient's history, the importance of neuraxial anesthesia to the patient, and the importance of the number of daily injections. Most patients continue enoxaparin until a scheduled induction, and they are instructed to skip their dose if labor symptoms begin. Patients at high risk for preterm delivery can be transitioned to heparin earlier than 36 weeks.

\section{References}

1. Creanga AA, Syverson C, Seed K, et al. Pregnancy-related mortality in the United States, 2011-2013. Obstet Gynecol. 2017;130:366-373. doi: 10.1097/AOG.0000000000002114

2. Kourlaba G, Relakis J, Kontodimas S, et al. A systematic review and meta-analysis of the epidemiology and burden of venous thromboembolism among pregnant women. Int J Gynaecol Obstet. 2016;132:4-10. doi: 10.1016/j.ijgo.2015.06.054.

3. Sultan AA, West J, Tata LJ, et al. Risk of first venous thromboembolism in and around pregnancy: a populationbased cohort study. Br J Haematol. 2012;156:366-373. doi: 10.1111/j.1365-2141.2011.08956.x.

4. American College of Obstetricians and Gynecologists. Council on Patient Safety in Women's Health Care: maternal venous thromboembolism (+AIM). 2015. https://safehealthcareforeverywoman.org/council/patient-safety-bundles/ maternal-safety-bundles/maternal-venous-thromboembolism-aim/. Accessed February 26, 2021.

5. Urato AC, Abi-Jaoude E, Abramson J, et al. National Partnership for Maternal Safety: consensus bundle on venous thromboembolism. Obstet Gynecol. 2019;134:1115-1117. doi: 10.1097/AOG.0000000000003540.

6. American College of Obstetricians and Gynecologists' Committee on Practice Bulletins-Obstetrics. ACOG practice bulletin no. 196: thromboembolism in pregnancy. Obstet Gynecol. 2018;132:el-el7. doi: 10.1097/AOG.0000000000002706.

7. Bates SM, Rajasekhar A, Middeldorp S, et al. American Society of Hematology 2018 guidelines for management of venous thromboembolism: venous thromboembolism in the context of pregnancy. Blood Adv. 2018;2:3317-3359. doi: 10.1182/bloodadvances.2018024802.

8. American College of Obstetricians and Gynecologists' Committee on Practice Bulletins-Obstetrics. ACOG practice bulletin no. 197: inherited thrombophilias in pregnancy. Obstet Gynecol. 2018;132:e18-e34. doi: 10.1097/ AOG.0000000000002703.

9. Bates SM, Greer IA, Middeldorp S, et al. VTE, thrombophilia, antithrombotic therapy, and pregnancy: Antithrombotic Therapy and Prevention of Thrombosis, 9th ed: American 
College of Chest Physicians evidence-based clinical practice guidelines. Chest. 2012;141(2, suppl):e691S-e736S. doi: 10.1378/chest.11-2300.

10. Lamont MC, McDermott C, Thomson AJ, et al. United Kingdom recommendations for obstetric venous thromboembolism prophylaxis: evidence and rationale. Semin Perinatol. 2019;43:222-228. doi: 10.1053/j.semperi.2019.03.008.

11. National Institutes of Health. COVID-19 Treatment Guidelines Panel. Coronavirus disease 2019 (COVID-19) treatment guidelines. https://www.covid19treatmentguidelines.nih. gov/. Accessed February 26, 2021.

12. Society for Maternal-Fetal Medicine (SMFM); Pacheco LD, Saade G, Metz TD. Society for Maternal-Fetal Medicine Consult Series \#51: thromboembolism prophylaxis for cesarean delivery. Am J Obstet Gynecol. 2020;223:B11-B17. doi: 10.1016/j.ajog.2020.04.032

13. Overcash RT, Somers AT, LaCoursiere DY. Enoxaparin dosing after cesarean delivery in morbidly obese women. Obstet Gynecol. 2015;125:1371-1376. doi: 10.1097/ AOG.0000000000000873.
14. Hiscock RJ, Casey E, Simmons SW, et al. Peak plasma antiXa levels after first and third doses of enoxaparin in women receiving weight-based thromboprophylaxis following caesarean section: a prospective cohort study. Int J Obstet Anesth. 2013;22:280-288. doi: 10.1016/j.ijoa.2013.05.008.

15. Palmerola KL, D'Alton ME, Brock CO, et al. A comparison of recommendations for pharmacologic thromboembolism prophylaxis after caesarean delivery from three major guidelines. BJOG. 2016;123:2157-2162. doi: 10.1111/14710528.13706.

16. Tran JP, Stribling SS, Ibezim UC, et al. Performance of risk assessment models for peripartum thromboprophylaxis. Reprod Sci. 2019;26:1243-1248. doi: 10.1177/1933719118813197.

17. Leffert L, Butwick A, Carvalho B, et al; members of the SOAP VTE Taskforce. The Society for Obstetric Anesthesia and Perinatology consensus statement on the anesthetic management of pregnant and postpartum women receiving thromboprophylaxis or higher dose anticoagulants. Anesth Analg 2018;126:928-944. doi: 10.1213/ANE.0000000000002530. 